\title{
THE INTERACTING SEYFERT 2 GALAXY UGC 3995A
}

\author{
D. DULTZIN-HACYAN \\ Instituto de Astronomía, UNAM \\ P. MARZIANI \\ Osservatorio Astronomico di Padova \\ AND \\ M. D' ONOFRIO \\ Dipartimento di Astronomia, Università di Padova
}

Direct observational evidence of interaction driven inflow of gas toward a Seyfert nucleus remains scant. UGC 3995 is one of the rare systems of galaxies that, at a first glance, offers direct evidence of this phenomenon: a bright filament appears to connect the bar of the spiral galaxy UGC 3995A (whose nucleus shows a Seyfert 2 spectrum) to the nucleus of a smaller spiral companion (UGC 3995B) whose radial velocity exceeds by only $30 \mathrm{~km} \mathrm{~s}^{-1}$ that of UGC 3995A. Narrow (redshifted $\mathrm{H} \alpha$ ) and broad band (R, B) images of UGC 3995, along with long slit spectra in the $\mathrm{H} \alpha$ region were collected at the $2.1 \mathrm{~m}$ telescope of the Observatorio Astronómico Nacional at San Pedro Mártir (SPM), México, in January-February 1995. From the B-R color map we infer that UGC 3995B is partially obscuring UGC 3995A. It is likely that a conspiracy of chance alignments and obscuration is giving the bar of UGC 3995A a disrupted appearance suggestive of stripping. The fairly regular profile of the $\mathrm{HI} 21 \mathrm{~cm}$ line suggests that the neutral hydrogen disks of both UGC 3995A and B have not yet undergone strong perturbations. However, while on the eastern side of UGC 3995A we observe HII region gas approximately following the rotation of the HI gas, on the western side we may be observing shock-heated "minority" gas whose motion is not exclusively rotational. We conclude that most of the morphological peculiarities observed in UGC 3995 cannot be easily related to infall of gas toward the Seyfert nucleus. Nevertheless, the kinematics of ionized gas in the western part of the disk of UGC 3995A has been affected by the intruder, probably because UGC 3995B is already physically in contact with UGC 3995A. 